\title{
Inter-Plaquette Correlations in U(1) Hamiltonian Lattice Gauge Systems
}

\author{
N. J. Davidson and R. F. Bishop* \\ Department of Mathematics, UMIST (University of Manchester Institute of Science and Technology), \\ P.O. Box 88, Manchester M60 1QD, United Kingdom
}

The coupled-cluster method is a very powerful method of quantum many-body theory which has recently been applied to Hamiltonian formulations of lattice gauge field theories. Previous calculations in the $U(1)$ model in $2+1$ dimensions have employed a local approximation scheme in which only correlations between adjacent plaquettes are taken into account. Here, we extend these calculations to include longer-ranged two-plaquette correlations, and also apply the local approximation scheme to the $U(1)$ model in $3+1$ dimensions.

\section{INTRODUCTION}

The Hamiltonian formulation of lattice gauge theories can be advantageous since it allows the application of the techniques of many-body theory to their solution. In particular, recent years have seen the application of two of the most powerful techniques of quantum many-body theory, namely the method of correlated basis functions (CBF) [1] and the coupled-cluster method (CCM) [2], to such problems [3-6].

In previous work on the $U(1)$ model $[3,4,6]$, only correlations between neighbouring plaquettes were considered. Here we examine the effect of including longer ranged two-plaquette correlations in the CCM approach to the $U(1)$ model in $2+1$ dimensions (2+1 D). We also apply the CCM to the calculation of the ground-state energy of the $U(1)$ model in the more realistic case of $3+1$ $D$. The generalisation from $2+1 \mathrm{D}$ to $3+1 \mathrm{D}$ is by no means trivial; while the former case does not possess a phase transition, several calculations suggest the presence of a (probably) second-order transition in the $3+1 \mathrm{D}$ case [7-9].

\section{THE $U(1)$ HAMILTONIAN}

The (scaled) Hamiltonian of $U(1)$ lattice gauge theory is usually written as

$H=-\frac{1}{2} \sum_{l} \frac{\partial^{2}}{\partial A_{l}^{2}}+\lambda \sum_{p}\left(1-\cos B_{p}\right)$,

*This work is supported by a research grant from SERC (Great Britain). where the first summation runs over all links of the lattice $l$ and the second over all plaquettes $p$. The coupling constant $\lambda$ lies in the interval $[0, \infty)$, with the limits $\lambda \rightarrow 0$ and $\lambda \rightarrow \infty$ being referred to as the strong and weak coupling limits respectively. $A_{l}$ is the vector potential defined on the link $l$, while the magnetic field $B_{p}$ is its lattice curl, namely a plaquette variable defined by the values of $A_{l}$ on the four links which comprise the plaquette.

It is now possible, by simple application of the chain rule of differentiation, to write the first summation in (1), the electric part of the Hamiltonian, in terms of the plaquette variables $\left\{B_{p}\right\}$. The Hamiltonian and its eigenfunctions are then expressed in explicitly gauge-invariant form:

$$
\begin{aligned}
H=\sum_{p}[ & -2 \frac{\partial^{2}}{\partial B_{p}^{2}}+\frac{1}{2} \sum_{v} \mathcal{P}^{(v)} \frac{\partial^{2}}{\partial B_{p} \partial B_{p+v}} \\
& \left.+\lambda\left(1-\cos B_{p}\right)\right]
\end{aligned}
$$

where plaquettes $p$ and $p+v$ have a link in common, and $\mathcal{P}^{(v)}$ is +1 if the common link appears with opposite sign in $B_{p}$ and $B_{p+v}$, and -1 otherwise. For $2+1 \mathrm{D}, \mathcal{P}^{(v)}$ is always +1 .

\section{CCM TREATMENT OF $U(1)$ LAT- TICE GAUGE THEORY}

The CCM assumes an exponential ansatz for the exact many-body wavefunction $|\Psi\rangle$ of a system, $|\Psi\rangle=\exp (S)|\Phi\rangle$, where $S$ is the correlation operator and $|\Phi\rangle$ is the reference state relative to which the correlations are measured. For our 
reference state, we consider the strong coupling limit $(\lambda \rightarrow 0)$, whence the electric vacuum state is simply a constant. The simplest gauge-invariant excitations are then given by the trigonometric functions $\cos m B$ and $\sin m B$ with $m$, the mode index or winding number, a non-negative integer.

The correlation operator $S$ is taken to be a function of the set of plaquette variables $\left\{B_{p}\right\}$ to ensure that we remain in the uncharged gaugeinvariant sector. We divide $S$ into a sum over $k$-body correlation operators (partitions) $S_{k}$

$$
S\left(\left\{B_{p}\right\}\right)=\sum_{k=1}^{N_{p}} S_{k}\left(\left\{B_{p}\right\}\right),
$$

where $N_{p}$ is the total number of plaquettes in the lattice and the $S_{k}$ are given by

$$
\begin{aligned}
S_{1}= & \sum_{n=1}^{\infty} \sum_{p=1}^{N_{p}} \mathcal{S}_{p}(n) \cos n B_{p} \\
S_{2}= & \frac{1}{2 !} \sum_{n_{1}, n_{2}=1}^{\infty} \sum_{p_{1}, p_{2}=1}^{N_{p}} \\
& {\left[\mathcal{S}_{p_{1} p_{2}}^{(1)}\left(n_{1}, n_{2}\right) \cos n_{1} B_{p_{1}} \cos n_{2} B_{p_{2}}\right.} \\
+ & \left.\mathcal{S}_{p_{1} p_{2}}^{(2)}\left(n_{1}, n_{2}\right) \sin n_{1} B_{p_{1}} \sin n_{2} B_{p_{2}}\right],
\end{aligned}
$$

with obvious generalization for $k>2$. The prime on the plaquette summation in $S_{2}$ indicates the omission of the term $p_{1}=p_{2}$. The coefficents $\mathcal{S}_{p_{1} \ldots}\left(n_{1}, \ldots\right)$ are determined from the CCM equations $[3,4,6]$. The construction of $|\Psi\rangle$ in the above way ensures that the ground state has positive parity under the simultaneous interchange of sign of all the $\left\{B_{p}\right\}$.

The last aspect of formalism which we require is the definition of an inner product. It is convenient, for reasons which we discuss below, to rewrite functions of the plaquette variables in terms of the link variables using the definition of the plaquette. For wavefunctions $\left\langle\tilde{g}\left(\left\{A_{l}\right\}\right)\right|$ and $\left|f\left(\left\{A_{l}\right\}\right)\right\rangle$, the inner product is defined as

$$
\langle\tilde{g} \mid f\rangle=\int_{-\pi}^{\pi}\left[\prod_{l=1}^{N_{l}} \frac{d A_{l}}{2 \pi}\right] \tilde{g} f .
$$

For the case of $2+1 \mathrm{D}$, this prescription is entirely equivalent to integrating over the $B_{p}$, whereas the possibility of constructing closed structures on the the $3+1 \mathrm{D}$ lattice requires the integration over the links $A_{l}$ to ensure that the Bianchi identities are satisfied.

To proceed further, we have to implement some approximations to make the problem tractable. These approximations are discussed in detail in refs. [3] and [4], but can be summarized as follows: The SUBn scheme keeps only partitions $S_{k}$ for $k$ smaller than $n$. The LSUB $n$ scheme keeps only those terms in SUBn which correspond to correlations between plaquettes which lie within a region on the lattice defined by $n$ contiguous plaquettes in all possible configurations. In this paper, we will concentrate on two-plaquette correlations, and define another SUB2 sub-truncation scheme, namely the SUB2$m$ scheme, in which only correlations between two plaquettes which are $m$-th nearest neighbours or closer are included. We note that LSUB2 is the same as SUB2-1, so that SUB2- $m$ can be viewed as interpolatating between LSUB2 and full SUB2. Furthermore, the mode indices can be restricted so that the sum of the mode indices in those terms retained in $S$ does not exceed a fixed value, say $k$. These sub-approximations are designated $\mathrm{SUB} n$ $m(k)$ and $\operatorname{LSUB} n(k)$.

\section{RESULTS}

In Figure 1 we show the ground-state energy per plaquette $E_{g} / N_{p}$ from SUB2-m(4) calculations for $2+1 \mathrm{D}$ for $0 \leq \lambda \leq 4$, from which it is clear that the local $(m=1)$ approximation is good for couplings well into the weak-coupling regime. In Figure 2, we show $E_{g} / N_{p}$ for $3+1 \mathrm{D}$ for several LSUB2 approximations. The convergence with $k$ of the LSUB2 $(k)$ sequence is good for $\lambda<2$, but appears to break down for larger couplings. This is in contrast to the LSUB2 $(k) 2+1$ $D$ results, where the sequence converges well for even very high values of $\lambda$ provided one chooses $k$ large enough [4]. The CCM results for $\lambda<0.6$ compare very well with Monte Carlo $[7,8]$ and loop calculus [9] results. For larger couplings, the Monte Carlo and loop calculus results suggest the presence of a second-order phase transition at $\lambda \approx 0.65$, which the LSUB2 results fail to 


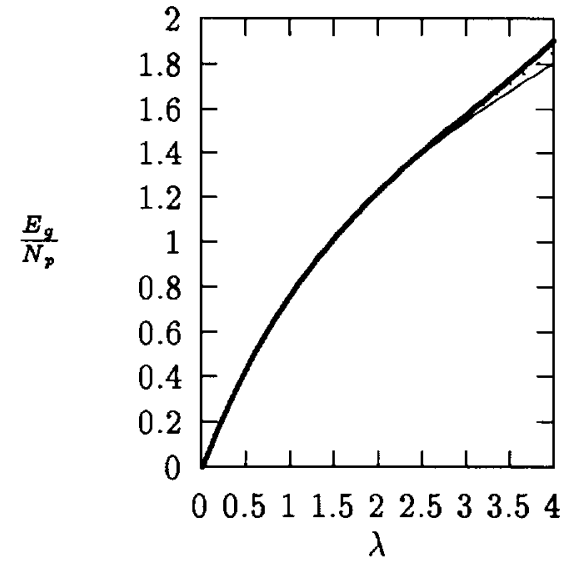

Figure 1. Ground-state energy per plaquette in $2+1$ dimensions (square lattice) for SUB21(4) (thin line), SUB2-2(4) (dots) and SUB2-3(4) (thick line).

reproduce. The presence of this phase transition is most likely the cause of the breakdown in the convergence of the LSUB2 results.

\section{CONCLUSIONS}

The CCM provides a microscopic approach to lattice gauge theory which is manifestly nonperturbative. As such, it allows for calculations to be performed well beyond the limits of ordinary perturbation theory without having to resort to ad-hoc resummation techniques such as Padé approximants

The results presented suggest that the loworder local approximation schemes work very well for the $U(1)$ model in $2+1 \mathrm{D}$ up to quite large couplings. In the case of $3+1 \mathrm{D}$, the (probable) presence of a phase transition leads to a breakdown in the convergence of the LSUB2 approximation, and more work is obviously required to determine the position of this transition. An improvement in the model state may be a comparatively simple way forward in this respect. In addition, other quantities, such as the string tension and the glueball mass, are of great interest

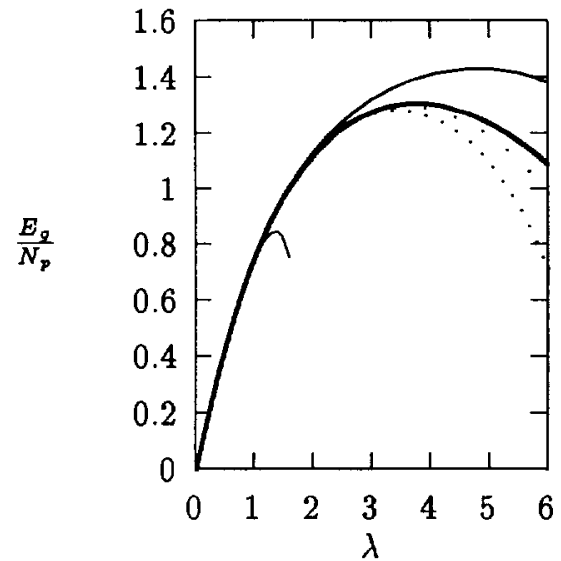

Figure 2. Ground-state energy per plaquette in $3+1$ dimensions (cubic lattice) for some LSUB2 $(k)$ approximations: $k=2$ (thin line), $k=3$ (medium line), $k=4$ (thick line), $k=5$ (close dots), $k=6$ (wide dots).

and are easily calculable within the CCM.

\section{REFERENCES}

1. J. W. Clark and E. Feenberg, Phys. Rev. 113 (1959) 388.

2. R. F. Bishop, Theor. Chim. Acta 80 (1991) 95 and references therein.

3. R. F. Bishop, A. S. Kendall, L. Y. Wong and Y. Xian, Phys. Rev. D 48 (1993) 887.

4. R. F. Bishop and Y. Xian, Acta Phys. Pol. B 3 (1993) 541.

5. C. H. Llewellyn-Smith and N. J. Watson, Phys. Lett. B302 (1993) 463.

6. R. F. Bishop and Y. Xian, Nucl. Phys. B (Proc. Suppl) 34 (1994) 811.

7. S. A. Chin, J. W. Negele and S. E. Koonin, Ann. Phys 157 (1984) 140.

8. C. J. Hamer and M. Aydin, Phys. Rev. D 43 (1991) 4080.

9. J. M. Aroca and H. Fort, Phys. Lett. B299 (1993) 305. 